\section{Mortalidade relacionada à hanseníase no Estado do Piauí, Brasil: tendências temporais e padrões espaciais, $2000-2015$}

\author{
Leprosy-related mortality in the State of Piauí, \\ Brazil: time trends and spatial patterns, \\ 2000-2015
}

\section{Mortalidad relacionada con la hanseniasis en el Estado de Piauí, Brasil: tendencias temporales y patrones espaciales, 2000-2015}

Olivia Dias de Araújo 1

Anderson Fuentes Ferreira 2

Telma Maria Evangelista de Araújo 1

Laila Caroline Leme da Silva 1

Walquirya Maria Pimentel Santos Lopes 1

Érica Alencar Rodrigues Neri 1

Jonas Alves Cardoso 1

Joelma Maria Costa 1

Edmércia Holanda Moura 1

Sandra Marina Gonçalves Bezerra 3

Michelle Santos Macêdo 1

Alberto Novaes Ramos Jr. 2

doi: 10.1590/0102-311X00093919

\section{Correspondência}

O. D. Araújo

Universidade Federal do Piauí.

Campus Universitário Ministro Petrônio Portela, Teresina, PI 64049-550, Brasil.

oliviaenf@ufpi.edu.br

1 Universidade Federal do Piauí, Teresina, Brasil. 2 Universidade Federal do Ceará, Fortaleza, Brasil.

3 Universidade Estadual do Piauí, Teresina, Brasil. mortalidade por regressão Joinpoint e análise espacial, usando os 224 nicípios como unidade geográfica. Dos 245.413 óbitos identificados, a hanseníase foi identificada em 324 declarações, 135 (41,7\%) como causa básica de óbito e 189 (58,3\%) como associada. Os maiores coeficientes de mortalidade relacionados à hanseníase foram observados entre homens (risco relativo - $R R=2,38$; IC95\%: 1,87; 3,03), idosos ( $R R=10,52$; IC95\%: 7,16; 15,46), cor parda $(R R=2,22$; IC95\%: 1,47; 3,35) e residentes do interior do estado $(R R=5,72 ;$ IC95\%: 4,54; 7,21). O coeficiente bruto de mortalidade relacionado à hanseníase apresentou incremento significativo entre idosos (70 anos), raça/cor parda, em cidades com menos de 20 mil habitantes e região Meio-norte, mas não significativo para o Estado do Piauí. A distribuição espacial pelos coeficientes de mortalidade ajustada por idade foi heterogênea nos municípios, concentrando altos coeficientes de mortalidade no norte do estado, próximo ao litoral. Verificou-se padrão de aumento dos coeficientes de mortalidade suavizados no decorrer dos quadriênios do estudo, concentrando altos coeficientes nas regiões Meio-norte e Semiárido. A mortalidade por hanseníase é espacialmente heterogênea e crescente ao longo dos anos. Ressalta-se a importância de potencializar ações integradas de vigilância e atenção à saúde.

Hanseníase; Mortalidade; Análise Espacial; Estudos de Séries Temporais 


\section{Introdução}

A hanseníase tem uma base neurológica de expressão clínica, com acometimento de nervos periféricos e pele 1. Entretanto, pode acometer outros órgãos e tecidos, incluindo mucosa do trato respiratório alto, vísceras abdominais, linfonodos, medula óssea, testículos, músculos e ossos 1,2. Causada pelo Mycobacterium leprae, é considerada uma doença crônica, porém curável, apresentando manifestações clínicas, associando diferentes respostas imunológicas à infecção bacteriana 3 .

O Brasil ainda detém o maior número de casos de hanseníase nas Américas e o segundo maior do mundo, após a Índia 4, o que amplia sua responsabilidade pela geração de evidências com vistas ao controle. Em 2018, foram diagnosticados 28.660 casos novos, com coeficiente de detecção geral de 13,74 casos por 100 mil habitantes (Ministério da Saúde. http://sage.saude.gov.br/, acessado em 25/Set/2019; Ministério da Saúde. http://www.saude.gov.br/saude-de-a-z/hanseniase, acessado em 25/Set/2019).

A Região Nordeste do país é a terceira com maior coeficiente de detecção geral, com 20,36/100 mil habitantes (Ministério da Saúde. http://www.saude.gov.br/saude-de-a-z/hanseniase, acessado em 25/Set/2019), o que caracteriza presença de alta endemicidade para a doença 5. Neste cenário, o Estado do Piauí contribuiu com 1.021 novos casos em 2018 (31,27 casos por 100 mil habitantes) (Ministério da Saúde. http://www.saude.gov.br/saude-de-a-z/hanseniase, acessado em 25/ Set/2019), dos quais 60 foram detectados em pessoas com menos de 15 anos de idade (8,9 casos por 100 mil habitantes) 6 .

A despeito de sua relevância, a mortalidade por hanseníase tem sido uma temática pouco estudada em países endêmicos 7,8. Os óbitos por hanseníase estão relacionados em grande parte ao manejo clínico de complicações sistêmicas da doença, incluindo incapacidade física e deformidades, bem como à ocorrência de infecções secundárias, episódios reacionais, além de eventos adversos à poliquimioterapia (PQT) e ao tratamento de episódios reacionais hansênicos 7,9.

Entre as doenças negligenciadas no Brasil, com carga de doença considerável 10, a hanseníase apresenta destaque, seja em análises considerando o óbito por causa básica ${ }^{9}$, seja o óbito por causa múltipla (básica e associada) 11. As lacunas que persistem nesta temática dificultam analisar sua real magnitude e expressão clínico-epidemiológica 2,9.

O conhecimento do real impacto dos óbitos para a compreensão da carga da hanseníase como condição crônica contribui para implementar estratégias para atenção integral, a partir da sensibilização de gestores e profissionais da rede de atenção à saúde, a fim de fornecer evidências para o controle da doença. Nesta perspectiva, este estudo teve por objetivo analisar os padrões espaciais e as tendências temporais da mortalidade relacionada à hanseníase no Estado do Piauí, Região Nordeste do Brasil, no período de 2000 a 2015.

\section{Métodos}

\section{Desenho do estudo}

Estudo ecológico misto, com análises espacial e temporal, de base populacional e âmbito estadual, realizado no Estado do Piauí. Utilizou-se como unidade de análise o conjunto de 224 municípios que integram as quatro macrorregiões de saúde do estado.

\section{Área de estudo}

O Piauí é o 19o mais populoso estado brasileiro, com uma população estimada para 2018 de 3.264 .531 milhões de habitantes. Em extensão, é o 11o maior estado do país, com densidade populacional de 12,40 habitantes $/ \mathrm{km}^{2}$. A maior parte dos municípios apresenta pequeno porte ( $<50$ mil habitantes) e aproximadamente $34,2 \%$ de sua população vive em áreas rurais (Figura 1) (Instituto Brasileiro de Geografia e Estatística. https://cidades.ibge.gov.br/brasil/pi/panorama, acessado em 20/Jan/2019). 


\section{Figura 1}

Regiões de saúde do Estado do Piauí, Região Nordeste do Brasil.

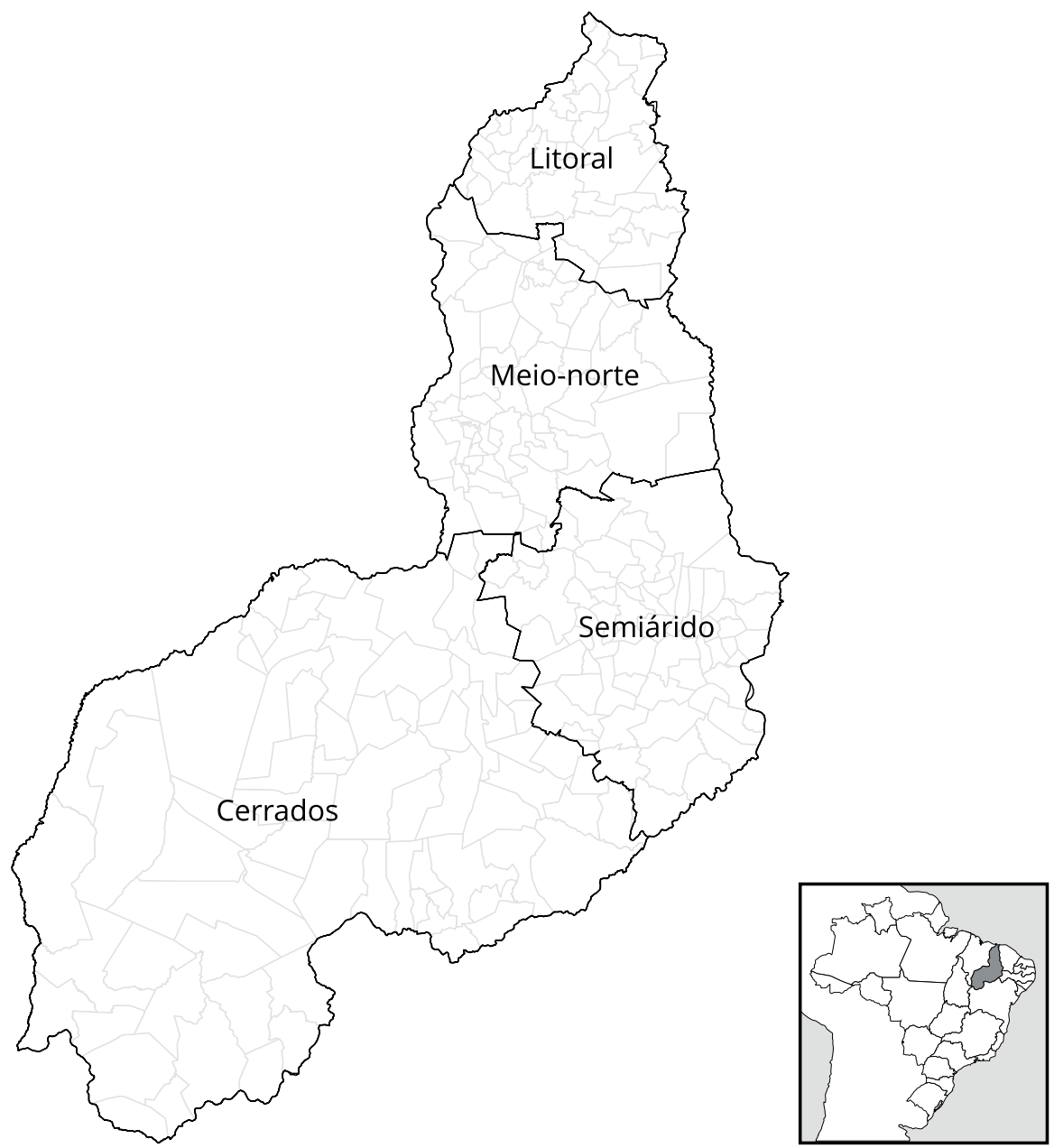

\section{Fonte de dados}

Foram incluídos todos os óbitos ocorridos no Estado do Piauí no período de 2000 a 2015, sendo consideradas todas as declarações de óbito em que a hanseníase estava mencionada como causa básica ou associada de morte (denominadas causas múltiplas de morte) 12.

As causas de morte relacionadas à hanseníase foram identificadas de acordo com a 10a revisão da Classificação Internacional de Doenças (CID-10): A30 ("Hanseníase [doença de Hansen] [lepra]"); A30.0: "Hanseníase [lepra] indeterminada”, A30.1: "Hanseníase [lepra] tuberculoide", A30.2: "Hanseníase [lepra] tuberculoide borderline”, A30.3: "Hanseníase [lepra] dimorfa”, A30.4: "Hanseníase [lepra] lepromatosa borderline", A30.5: "Hanseníase [lepra] lepromatosa”, A30.8: "Outras formas de hanseníase [lepra]" e A30.9: "Hanseníase [lepra] não especificada” e B92: “Sequelas de hanseníase [lepra]” 12. 
Os dados de mortalidade do Estado do Piauí foram obtidos a partir do Sistema de Informações sobre Mortalidade (SIM) do Ministério da Saúde. Esses dados são de domínio público e estão disponíveis no site do Departamento de Informática do Sistema Único de Saúde (DATASUS. http://www2. datasus.gov.br/DATASUS/index.php?area=0205, acessado em 20/Fev/2019).

O SIM contém dados referentes a todas as declarações de óbitos preenchidas por profissionais médicos. Nessa declaração, devem constar as causas básicas e associadas de morte, além de variáveis relacionadas a sexo/gênero, idade, raça/cor, nível educacional, estado civil, local de residência ou ocorrência de morte e causas de morte (causa básica ou associada de morte), dentre outras. Uma vez preenchida, cabe às secretarias municipais e estaduais de saúde a análise para codificação e a entrada de dados no sistema (DATASUS. http://tabnet.datasus.gov.br/cgi/sim/dados/cid10_indice.htm, acessado em 25/Fev/2019).

Os dados populacionais foram obtidos diretamente no Instituto Brasileiro de Geografia e Estatística, com base nos censos nacionais de população (2000 e 2010) e nas estimativas de população (2001 a 2009 e 2011 a 2015) (Instituto Brasileiro de Geografia e Estatística. https://cidades.ibge.gov.br/brasil/ pi/panorama, acessado em 20/Jan/2019).

\section{Características epidemiológicas}

Procedeu-se ao cálculo dos coeficientes brutos de mortalidade (com seus respectivos intervalos de 95\% de confiança [IC95\%]), estratificados por sexo/gênero (masculino e feminino), faixa etária $(<15,15-29,30-39,40-49,50-59,60-69$ e $\geq 70)$, estado civil (casado, desquitado ou separado judicialmente ou divorciado, solteiro e viúvo), raça/cor (amarela, branca [caucasiana], preta [afro-brasileira/ afrodescendente] e parda [raça mista/pardo brasileira]), residência em capital (sim e não), regiões de saúde (regiões sanitárias do Estado do Piauí: Cerrados, Litoral, Meio-norte e Semiárido) (Figura 1) e porte do município (pequeno porte I: até 20.000 habitantes; pequeno porte II: de 20.001 a 50.000 habitantes; médio porte: de 50.001 a 100.000 habitantes; grande porte: > 100.001 habitantes). O cálculo foi realizado dividindo-se a média do número de óbitos relacionados à hanseníase, por ano, pela população sob risco (expressa por 100 mil habitantes), tendo como base a população do Censo Demográfico de 2010.

Para determinar as diferenças entre os grupos, estimou-se o risco relativo (RR) com seus respectivos IC95\%. Utilizou-se o teste qui-quadrado de Pearson para cálculo da significância estatística de $5 \%$. As categorias de referência para o cálculo do RR se basearam em estudos de mortalidade $8,9,11$. As análises estatísticas foram realizadas utilizando o software Stata versão 11.2 (https://www.stata.com).

\section{Analise espacial}

Para análise da distribuição espacial e dos padrões espaciais de mortalidade relacionada à hanseníase, utilizaram-se como unidade de análise geográfica os municípios de residência do Piauí ( $\mathrm{n}=$ 224, divisão territorial de 2013). Foram excluídos os óbitos em que o município de residência era desconhecido.

Procedeu-se ao cálculo dos coeficientes médios de mortalidade ajustados por idade relacionada à hanseníase (por 100 mil habitantes) no nível municipal para o período de 16 anos de estudo, dividindo-se em quadriênios (2000-2003, 2004-2007, 2008-2011, 2012-2015) e no período total. Os coeficientes ajustados por idade foram calculados pelo método direto de padronização, utilizando-se a população brasileira do Censo Demográfico de 2010 como padrão. As categorias de idade empregadas no processo de padronização e no cálculo dos coeficientes foram: < 15, 15-29, 30-39, 40-49, 50-59, 60-69 e $\geq 70$ anos. Municípios sem o registro de óbitos por hanseníase apresentaram coeficiente de mortalidade igual a "zero". Para redução da flutuação aleatória causada por eventos raros, municípios com população pequena e/ou subnotificação, calcularam-se os coeficientes de mortalidade suavizados (por 100 mil habitantes) por meio do método Bayesiano empírico local.

Os softwares ArcGIS versão 9.3 (http://www.esri.com/software/arcgis/index.html) e TerraView versão 4.2.2 (http://www.dpi.inpe.br/terraview) foram utilizados para construção de mapas temáticos. 


\section{Tendência temporal}

Para análise da tendência temporal do coeficiente de mortalidade relacionado à hanseníase no Piauí, assim como da mortalidade proporcional, procedeu-se à regressão Joinpoint (por pontos de inflexão) de Poisson, utilizando-se o Joinpoint Regression Program versão 4.4.2 (http://surveillance.cancer. gov/joinpoint/). Essa técnica estatística regula o ajuste de uma série de linhas, bem como de seus pontos de inflexão, em uma escala logarítmica por meio do teste de tendências anuais. Com o intuito de obter a melhor linha de cada segmento, utilizou-se o método de permutação de Monte Carlo como teste da significância estatística (nível de $5 \%$ de significância).

A partir da definição dos segmentos, estimaram-se e testaram-se a variação percentual anual (annual percentual change - APC) e a variação percentual anual média (average annual percentual change - AAPC), com seus respectivos IC95\%. O resultado possibilitou demonstrar crescimento (valores de APC positivos), redução (valores de APC negativos) ou ausência significativa (valor de APC igual a zero) de tendência ao longo de toda a série histórica analisada (2000-2015).

Procedeu-se aos cálculos APC e AAPC (com seus períodos de tempo e IC95\%), estratificados por sexo/gênero, faixa etária, estado civil, raça/cor, regiões de saúde (regiões sanitárias do Estado do Piauí e municípios), residência em capital e porte do município. O cálculo foi realizado dividindo-se o número de óbitos relacionados à hanseníase, por ano, pela população sob risco (expressa por 100 mil habitantes).

\section{Considerações éticas}

O presente estudo foi baseado em dados secundários de mortalidade de acesso aberto ao público, de modo que não foi necessária a submissão para apreciação por um comitê de ética em pesquisa. $O$ estudo seguiu as recomendações do Conselho Nacional de Saúde (Resolução no 466 de 2012).

\section{Resultados}

\section{Características epidemiológicas}

Foram registrados 245.413 óbitos no Estado do Piauí em 16 anos de estudo. A hanseníase foi identificada em 324 declarações (mortalidade proporcional: 0,13\%; IC95\%: 0,11; 0,15), 135 (41,7\%) como causa básica de óbito e $189(58,3 \%)$ como causa associada. O número médio anual de óbitos relacionados à hanseníase foi de 20,25 por ano, com desvio padrão de 7,16 (IC95\%: 19,47; 21,03).

A causa do óbito mais frequente relacionada à hanseníase foi "hanseníase não especificada" (A30.9), tanto como causa básica quanto associada de morte (83,7\% dos óbitos [113/135] e 84,1\% [159/189] respectivamente). As sequelas de hanseníase (B92) foram identificadas em 8,9\% (12/135) como causa básica de morte e em 6,9\% (13/189) como causa associada.

O coeficiente médio anual de mortalidade relacionada à hanseníase foi de 0,65 óbitos/100 mil habitantes (IC95\%: 0,37-0,94). O risco relativo revelou-se maior quando a pessoa acometida pela hanseníase tinha as seguintes características: ser do sexo masculino ( $R R=2,38$; IC95\%: 1,87; 3,03) quando comparado ao sexo feminino; ter idade $\geq 70$ (RR = 10,52; IC95\%: 7,16; 15,46) quando comparada à faixa etária de 40-49; ser solteiro $(\mathrm{RR}=11,47$; IC95\%: 0,01; 0,81) em comparação com viúvos; raça/cor parda ( $R R=2,22$; IC95\%: 1,47; 3,35) quando comparada com a raça/cor branca; não residir na capital $(\mathrm{RR}=5,72$; IC95\%: 4,54; 7,21) em relação aos residentes em Teresina; e residir na região Meio-norte ( $R R=1,38$; IC95\%: 1,00; 1,88) quando comparado com residentes na região Cerrados. Os coeficientes de mortalidade mais altos foram de óbitos entre 60-69 anos de idade (22,3\% [72/324], coeficiente 3,00/100 mil habitantes), e com 70 anos ou mais de idade (37,2\% [120/324], coeficiente 6,50/100 mil habitantes) (Tabela 1). 
Tabela 1

Número, proporção de óbitos e coeficiente bruto de mortalidade (por 100 mil habitantes) por hanseníase segundo sexo, faixa etária, raça/cor e região de residência. Estado do Piauí, Brasil, 2000-2015.

\begin{tabular}{|c|c|c|c|c|c|c|c|}
\hline \multirow[t]{2}{*}{ Variáveis } & \multicolumn{2}{|c|}{ Óbitos } & \multicolumn{2}{|c|}{$\begin{array}{c}\text { Coeficiente de } \\
\text { mortalidade bruto * }\end{array}$} & \multirow[t]{2}{*}{ RR } & \multirow[t]{2}{*}{ IC95\% } & \multirow[t]{2}{*}{ Valor de $p$} \\
\hline & $\mathbf{n}$ & $\%$ & Coeficiente & IC95\% & & & \\
\hline Óbitos totais & 324 & 100,0 & 0,65 & 0,$37 ; 0,94$ & - & - & - \\
\hline \multicolumn{8}{|l|}{ Sexo/Gênero } \\
\hline Feminino & 94 & 29,0 & 0,38 & 0,$07 ; 0,70$ & Ref. & - & - \\
\hline Masculino & 230 & 71,0 & 0,92 & 0,$44 ; 1,39$ & 2,38 & 1,$87 ; 3,03$ & $<0,0001$ \\
\hline \multicolumn{8}{|l|}{ Grupo etário (anos) ** } \\
\hline$<15$ & 3 & 0,9 & 0,02 & 0,$00 ; 0,11$ & 0,03 & 0,$01 ; 0,11$ & $<0,0001$ \\
\hline $15-29$ & 15 & 4,6 & 0,10 & 0,$00 ; 0,31$ & 0,17 & 0,$09 ; 0,30$ & $<0,0001$ \\
\hline $30-39$ & 24 & 7,4 & 0,35 & 0,$00 ; 0,92$ & 0,57 & 0,$34 ; 0,97$ & 0,0388 \\
\hline $40-49$ & 33 & 10,2 & 0,62 & 0,$00 ; 1,46$ & Ref. & - & - \\
\hline $50-59$ & 56 & 17,3 & 1,48 & 0,$00 ; 3,03$ & 2,40 & $1,56.3,69$ & $<0,0001$ \\
\hline $60-69$ & 72 & 22,3 & 3,00 & 0,$23 ; 5,77$ & 4,86 & 3,$22 ; 7,33$ & $<0,0001$ \\
\hline$\geq 70$ & 120 & 37,2 & 6,50 & 1,$85 ; 11,16$ & 10,52 & 7,$16 ; 15,46$ & $<0,0001$ \\
\hline \multicolumn{8}{|l|}{ Estado civil ** } \\
\hline Casado & 161 & 53,3 & 1,19 & 0,$45 ; 1,92$ & 3,65 & 2,$80 ; 4,74$ & $<0,0001$ \\
\hline $\begin{array}{l}\text { Desquitado ou separado } \\
\text { judicialmente ou divorciado }\end{array}$ & 4 & 1,3 & 0,62 & 0,$31 ; 1,00$ & 1,89 & 0,$70 ; 5,16$ & 0,2116 \\
\hline Solteiro & 52 & 17,2 & 3,74 & 1,$40 ; 7,94$ & 11,47 & 8,$13 ; 16,20$ & $<0,0001$ \\
\hline Viúvo & 85 & 28,1 & 0,33 & 0,$05 ; 0,60$ & Ref. & - & - \\
\hline \multicolumn{8}{|l|}{ Raça/Cor ** } \\
\hline Amarela & 1 & 0,3 & 1,02 & 0,$76 ; 1,40$ & 2,51 & 0,$35 ; 18,09$ & 0,3626 \\
\hline Branca (caucasiana) & 57 & 18,9 & 0,41 & 0,$00 ; 0,83$ & Ref. & - & - \\
\hline $\begin{array}{l}\text { Preta (afro-brasileira/ } \\
\text { afrodescendente) }\end{array}$ & 206 & 68,4 & 0,60 & 0,$27 ; 0,93$ & 1,48 & 1,$10 ; 1,99$ & 0,0087 \\
\hline $\begin{array}{l}\text { Parda (raça mista/pardo } \\
\text { brasileira) }\end{array}$ & 37 & 12,3 & 0,90 & 0,$00 ; 2,07$ & 2,22 & 1,$47 ; 3,35$ & 0,0002 \\
\hline \multicolumn{8}{|l|}{ Residência na capital } \\
\hline Sim & 107 & 33,0 & 0,59 & 0,$28 ; 0,91$ & Ref. & - & \\
\hline Não & 217 & 67,0 & 0,83 & 0,$20 ; 1,45$ & 5,72 & 4,$54 ; 7,21$ & $<0,0001$ \\
\hline \multicolumn{8}{|l|}{ Região de residência } \\
\hline Cerrados & 51 & 15,7 & 0,56 & 0,$00 ; 1,17$ & Ref. & - & - \\
\hline Litoral & 55 & 17,0 & 0,53 & 0,$00 ; 1,09$ & 0,96 & 0,$65 ; 1,40$ & 0,8200 \\
\hline Meio-norte & 166 & 51,2 & 0,76 & 0,$30 ; 1,23$ & 1,38 & 1,$00 ; 1,88$ & 0,0466 \\
\hline Semiárido & 52 & 16,0 & 0,63 & 0,$00 ; 1,31$ & 1,12 & 0,$76 ; 1,65$ & 0,5519 \\
\hline \multicolumn{8}{|l|}{ Porte da cidade } \\
\hline Pequeno porte I & 119 & 36,7 & 0,58 & 0,$16 ; 0,99$ & 0,57 & 0,$39 ; 0,85$ & 0,0058 \\
\hline Pequeno porte II & 52 & 16,0 & 0,49 & 0,$00 ; 1,03$ & 0,49 & 0,$31 ; 0,76$ & 0,0016 \\
\hline Médio porte & 31 & 9,6 & 1,01 & 0,$00 ; 2,42$ & Ref. & - & - \\
\hline Grande porte & 122 & 37,7 & 0,80 & 0,$23 ; 1,37$ & 0,79 & 0,$54 ; 1,18$ & 0,2503 \\
\hline
\end{tabular}

IC95\%: intervalo de 95\% de confiança; RR: risco relativo.

* Coeficiente anual médio bruto (por 100 mil habitantes), calculado com base no número médio de mortes relacionadas com a hanseníase no numerador e o tamanho populacional no ano central do período estudado como denominador. Os dados populacionais sobre raça/cor foram obtidos a partir dos censos nacionais brasileiros (2000 e 2010). O tamanho da população em relação à raça/cor para o meio do período foi derivado das estimativas da Pesquisa Nacional por Amostra de Domicílios (PNAD).

** Dados não disponíveis em todos os casos (grupo etário: 1, estado civil: 22, raça/cor: 23). 


\section{Análise espacial}

Do total analisado, 46\% (103/224) dos municípios registraram pelo menos um óbito relacionado à hanseníase. Os coeficientes médios de mortalidade ajustados por idade entre municípios variaram de 0,00 a 14,92 óbitos/100 mil habitantes (Figura 2), enquanto os coeficientes de mortalidade suavizados por município variaram de 0,00 a 5,79 óbitos/100 mil habitantes (Figura 3).

\section{Figura 2}

Distribuição de óbitos relacionados à hanseníase, segundo códigos da 10ạ revisão da Classificação Internacional de Doenças (CID-10). Estado do Piauí, Brasil, 2000-2015. Mapas dos coeficientes ajustados por idade da mortalidade por 100 mil habitantes.
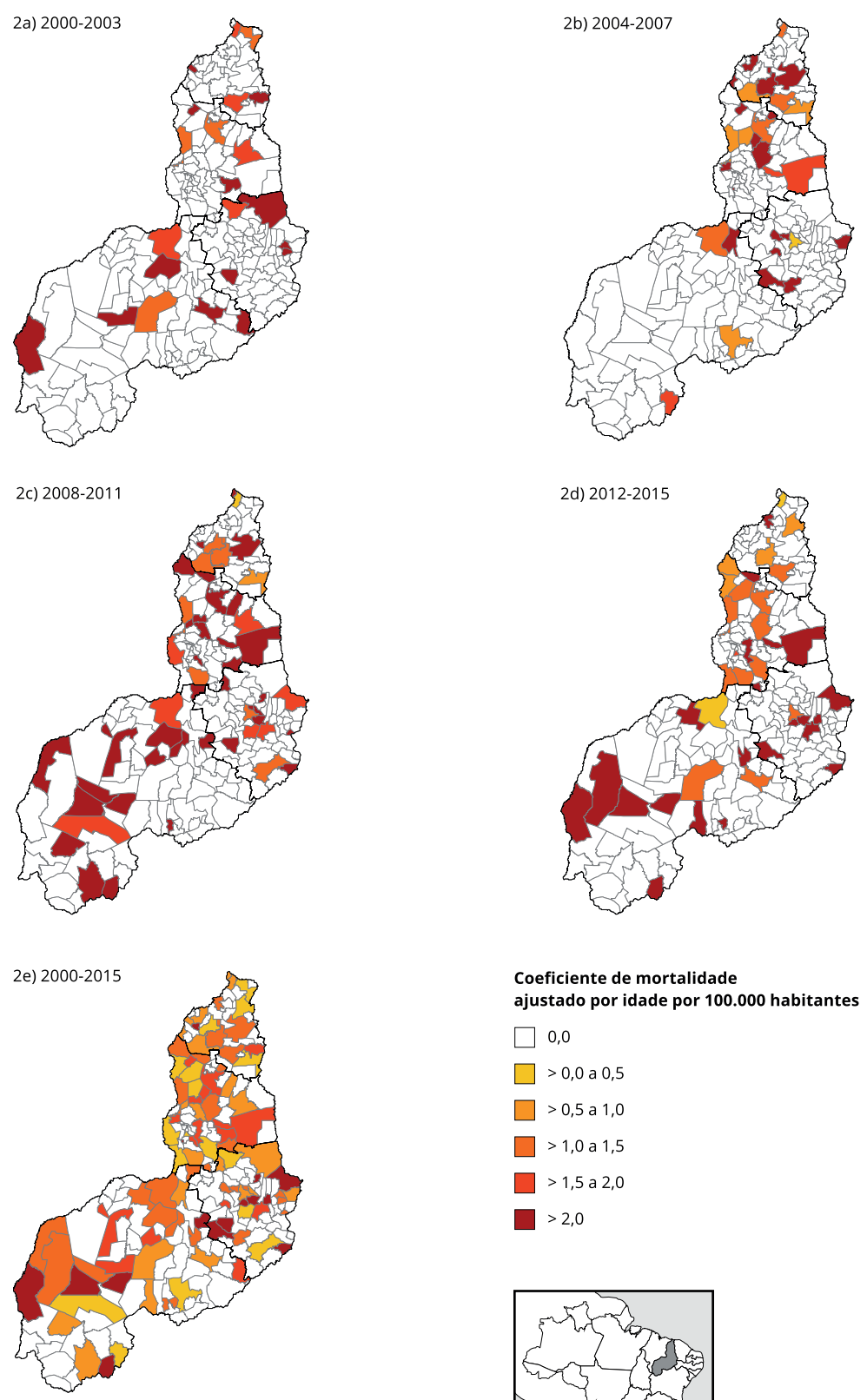

Coeficiente de mortalidade

ajustado por idade por 100.000 habitantes
0,0
$\square>0,0$ a 0,5
$>0,5$ a 1,0
$>1,0$ a 1,5
$>1,5$ a 2,0
$>2,0$

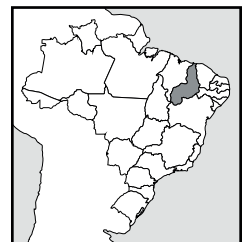


Figura 3

Distribuição de óbitos relacionados à hanseníase, segundo códigos da 10a revisão da Classificação Internacional de Doenças (CID-10). Estado do Piauí, Brasil, 2000-2015. Mapas dos coeficientes suavizados de mortalidade por 100 mil habitantes.
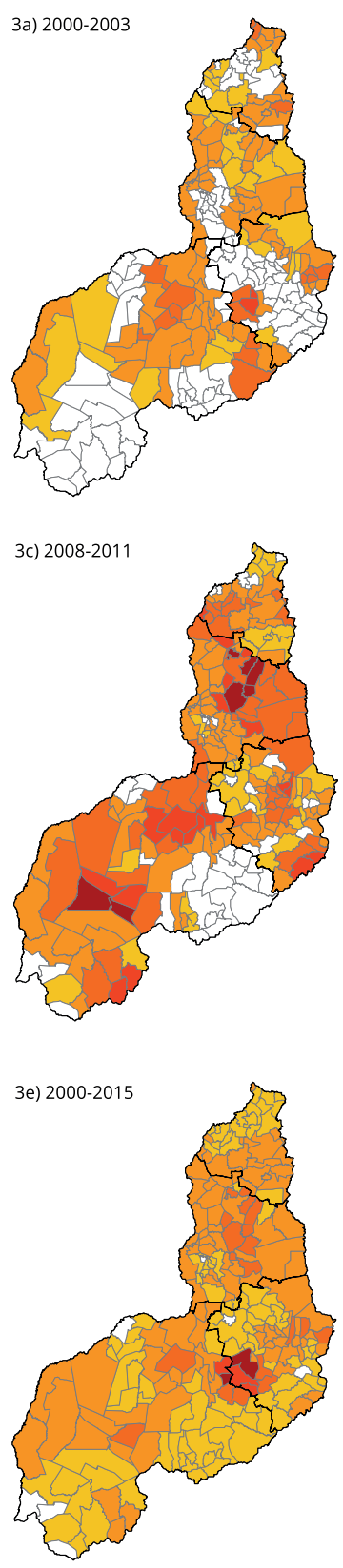
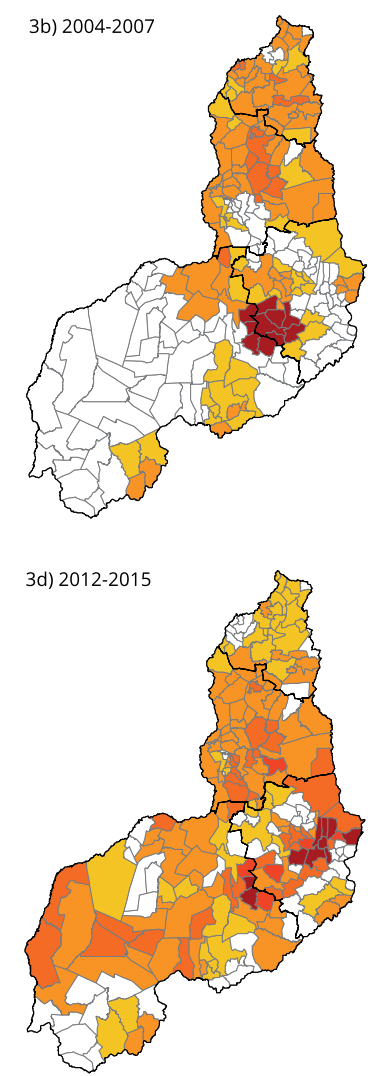

Coeficiente de mortalidade

suavizado por 100.000 habitantes

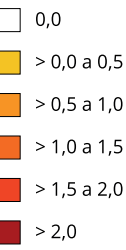

$>2,0$

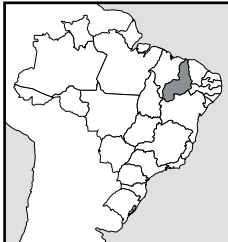

Foram identificados municípios com altos coeficientes de mortalidade relacionados à hanseníase (> 1,00 óbito/100 mil habitantes) no sul da região Litoral, leste da região Meio-norte, oeste da região Semiárido, e no norte e leste da região Cerrados (Figura 2). O método Bayesiano gerou coeficientes de mortalidade corrigidos mais estáveis, aumentando a abrangência de municípios de 2008-2011 e 2012-2015. Dentre as regiões, os coeficientes mais altos foram encontrados no centro da região 
Meio-norte, centro-sul da região Semiárido e leste da região Cerrados. Verificou-se ainda padrão de crescimento de coeficientes de mortalidade suavizados no decorrer dos quadriênios do estudo, concentrando altos coeficientes nas regiões Meio-norte e Semiárido do estado (Figura 3).

\section{Tendências temporais}

O coeficiente bruto de mortalidade relacionado à hanseníase apresentou incremento não significativo da tendência temporal (APC = 3,5; IC95\%: -0,2; 7,2), considerando toda a série histórica. Verificou-se incremento nas tendências nos coeficientes de mortalidade em: $\geq 70$ anos (APC = 6,4; IC95\%: 1,2; $11,8)$, raça/cor parda $(\mathrm{APC}=5,3 ; \mathrm{IC} 95 \%: 0,3 ; 10,5)$, na região Meio-norte $(\mathrm{APC}=4,1 ; \mathrm{IC} 95 \%: 0,2 ; 8,2)$ e em municípios com 20 mil habitantes ou menos (APC = 6,7; IC95\%: 0,3; 13,5) (Tabela 2).

\section{Tabela 2}

Análise de regressão por Joinpoint do coeficiente bruto relacionado à mortalidade associada à hanseníase, segundo sexo, faixa etária, raça/cor e região de residência. Estado do Piauí, Brasil, 2000-2015.

\begin{tabular}{|c|c|c|c|c|c|}
\hline \multirow[t]{2}{*}{ Indicador/Variáveis } & \multicolumn{3}{|c|}{ Tendência } & \multicolumn{2}{|c|}{ Período total } \\
\hline & Período & APC & IC95\% & AAPC & IC95\% \\
\hline Piauí - Total & $2000-2015$ & 3,5 & $-0,2 ; 7,2$ & 3,5 & $-0,2 ; 7,2$ \\
\hline \multicolumn{6}{|l|}{ Sexo/Gênero } \\
\hline Masculino & $2000-2015$ & 2,9 & $-3,6 ; 9,8$ & 2,9 & $-3,6 ; 9,8$ \\
\hline Feminino & $2000-2015$ & 3,5 & $-0,3 ; 7,5$ & 3,5 & $-0,3 ; 7,5$ \\
\hline \multicolumn{6}{|l|}{ Grupo etário (anos) } \\
\hline$<15$ & $2000-2015$ & $-0,5$ & $-3,2 ; 2,3$ & $-0,5$ & $-3,2 ; 2,3$ \\
\hline $15-29$ & $2000-2015$ & $-3,5$ & $-9,9 ; 3,2$ & $-3,5$ & $-9,9 ; 3,2$ \\
\hline $30-39$ & $2000-2015$ & $-5,1$ & $-10,6 ; 0,8$ & $-5,1$ & $-10,6 ; 0,8$ \\
\hline $40-49$ & $2000-2015$ & $-2,2$ & $-9,8 ; 6,0$ & $-2,2$ & $-9,8 ; 6,0$ \\
\hline $50-59$ & $2000-2015$ & $-0,5$ & $-8,0 ; 7,6$ & $-0,5$ & $-8,0 ; 7,6$ \\
\hline $60-69$ & $2000-2015$ & 0,9 & $-4,3 ; 6,5$ & 0,9 & $-4,3 ; 6,5$ \\
\hline$\geq 70$ & $2000-2015$ & 6,4 * & 1,$2 ; 11,8$ & 6,4 * & 1,$2 ; 11,8$ \\
\hline \multicolumn{6}{|l|}{ Estado civil } \\
\hline Casado(a) & $2000-2015$ & 3,1 & $-2,6 ; 9,2$ & 3,1 & $-2,6 ; 9,2$ \\
\hline Viúvo(a) & $2000-2015$ & 3,7 & $-2,0 ; 9,7$ & 3,7 & $-2,0 ; 9,7$ \\
\hline Solteiro(a) & $2000-2015$ & $-1,7$ & $-7,7 ; 4,7$ & $-1,7$ & $-7,7 ; 4,7$ \\
\hline \multicolumn{6}{|l|}{ Raça/Cor } \\
\hline Branca (caucasiana) & $2000-2015$ & 2,7 & $-2,2 ; 7,8$ & 2,7 & $-2,2 ; 7,8$ \\
\hline Preta (afro-brasileira/afrodescendente) & $2000-2015$ & $-3,7$ & $-8,8 ; 1,8$ & $-3,7$ & $-8,8 ; 1,8$ \\
\hline Parda (raça mista/pardo brasileira) & $2000-2015$ & 5,3 * & 0,$3 ; 10,5$ & 5,3 * & 0,$3 ; 10,5$ \\
\hline \multicolumn{6}{|l|}{ Região de residência } \\
\hline Cerrados & $2000-2015$ & 5,1 & $-3,2 ; 14,0$ & 5,1 & $-3,2 ; 14,0$ \\
\hline Litoral & $2000-2015$ & $-1,1$ & $-7,9 ; 6,3$ & $-1,1$ & $-7,9 ; 6,3$ \\
\hline Meio-norte & $2000-2015$ & 4,1 * & 0,$2 ; 8,2$ & 4,1 * & 0,$2 ; 8,2$ \\
\hline Semiárido & $2000-2015$ & 3,6 & $-2,9 ; 10,6$ & 3,6 & $-2,9 ; 10,6$ \\
\hline \multicolumn{6}{|l|}{ Residência na capital } \\
\hline Sim & $2000-2015$ & 2,3 & $-2,1 ; 6,9$ & 2,3 & $-2,1 ; 6,9$ \\
\hline Não & $2000-2015$ & 4,7 & $-0,3 ; 9,8$ & 4,7 & $-0,3 ; 9,8$ \\
\hline \multicolumn{6}{|l|}{ Porte da cidade } \\
\hline Pequeno porte I & $2000-2015$ & 6,7 * & 0,$3 ; 13,5$ & $6,7 *$ & 0,$3 ; 13,5$ \\
\hline Pequeno porte II & $2000-2015$ & 3,1 & $-4,5 ; 11,2$ & 3,1 & $-4,5 ; 11,2$ \\
\hline Médio porte & $2000-2015$ & 1,6 & $-3,4 ; 6,9$ & 1,6 & $-3,4 ; 6,9$ \\
\hline Grande porte & $2000-2015$ & 0,0 & $-4,0 ; 4,2$ & 0,0 & $-4,0 ; 4,2$ \\
\hline
\end{tabular}

APC: variação percentual média; AAPC: variação percentual anual média; IC95\%: intervalo de 95\% de confiança.

* Significantemente diferente de $0(p<0,05)$. 


\section{Discussão}

Pela primeira vez, este estudo apresenta uma análise dos óbitos relacionados à hanseníase ocorridos no Estado do Piauí em uma longa série histórica. A ocorrência de óbitos decorrentes da doença é relevante e se deu de maneira desigual nas regiões do estado ao longo dos 16 anos analisados. O estudo corrobora o cenário nacional 8 , por conta de a hanseníase ter sido mencionada tanto como causa básica como causa associada de óbito e também por ter causa de morte relacionada não especificada. A mortalidade relacionada à hanseníase foi maior entre homens, idosos, raça/cor parda e em regiões do interior do estado desfavorecidas econômica e socialmente.

A tendência de mortalidade relacionada à hanseníase não apresenta distribuição bem definida, visto que não ocorre da mesma forma em outros estados do Brasil 7. Estudo realizado no Estado da Bahia, no período de 1999-2014, apontou um aumento de óbitos por hanseníase em homens, corroborando a evidência de que o sexo masculino está mais susceptível ao adoecimento e morte por essa doença, trazendo um aspecto relevante de vulnerabilidade pela condição de gênero 7. No Brasil, por outro lado, o aumento se deu em ambos os sexos 8 .

A predominância de mortalidade associada à hanseníase entre homens pode estar relacionada a fatores comportamentais, modos culturais e pessoais de adoecimento e autocuidado, bem como da receptividade e preparo dos serviços de saúde no atendimento das demandas de saúde dos homens $8,13,14,15,16$.

Estudo realizado no nordeste da Colômbia, sobre fatores associados ao atraso no diagnóstico da hanseníase, constatou que os homens eram mais diagnosticados com hanseníase, com atraso médio no diagnóstico da doença de 33,5 meses, na forma multibacilar e com grau 2 de incapacidade física no momento do diagnóstico ${ }^{17}$.

Reconhece-se que as incapacidades e as deformidades físicas estão entre as complicações que podem gerar consequências irreparáveis às pessoas acometidas, que vão desde uma limitação funcional até um prognóstico indesejado 7,18, haja vista as incapacidades serem resultantes da perda de sensibilidade protetora e/ou deformidades que propiciam a ocorrência de reações hansênicas e lesões sucessivas que, aliadas a outros problemas, podem ocasionar o óbito 18,19,20.

$\mathrm{Na}$ avaliação do fator etário, o atual estudo apresentou um aumento de óbitos relacionados à hanseníase em idosos com mais de setenta anos, como observado em outros estudos de mortalidade por hanseníase 7,11,21. O adoecimento por hanseníase pode ser facilitado pelo processo de envelhecimento, em virtude do comprometimento das etapas naturais de defesa imunológica do idoso, tornando-o mais suscetível a desenvolver a doença e suas complicações, a exemplo das incapacidades físicas 22,23. Estudo realizado no Estado de Alagoas sobre o perfil epidemiológico de idosos com hanseníase apontou que os idosos entre 60-69 anos de idade, de classificação multibacilar e raça/cor parda, estão entre os mais atingidos pela hanseníase 24 .

Neste estudo, a população de raça/cor parda apresentou tendência de incremento de óbitos, semelhante ao encontrado em estudo realizado no Estado da Bahia 7. Grupos populacionais de cor parda e negra têm sido relatados como os mais acometidos pela doença, principalmente na classificação multibacilar 13. Entretanto, em estudo caso-controle realizado no Nordeste do Brasil, a ocorrência de hanseníase em populações de raça/cor negra e/ou preta não apresentou diferença significativa, quando comparadas com a raça/cor branca ${ }^{16}$. Apostar em mudanças culturais, econômicas e sociais pode contribuir para redução da carga de hanseníase em populações de pardos e/ou negros, bem como na população geral 15 .

Em todas as regiões do Piauí foram detectados óbitos pela doença, constatando-se um maior potencial de risco nas macrorregiões menos desenvolvidas. A região Meio-norte, onde se localiza a capital do Estado do Piauí, apresentou relevante mortalidade relacionada à hanseníase, embora a capital não tenha apresentado um aumento de óbitos pela doença. Esse incremento na região Meio-norte pode estar relacionado a municípios com vulnerabilidade social que dependem da capital do estado 25 .

Maiores coeficientes de prevalência da hanseníase têm sido observados em regiões mais pobres do Brasil, com padrões que refletem endemicidade para a doença em locais com expressiva desigualdade socioeconômica 26 . 
Os determinantes sociais permitem compreender a relação que o nível de organização da sociedade mantém com as condições de saúde da população, uma vez que podem influenciar a saúde das pessoas 27 . Consoante isso, já se sabe que fatores socioeconômicos aliados a fatores ambientais e comportamentais podem contribuir para que grupos populacionais desenvolvam hanseníase e suas complicações 8,16,28,29.

Ademais, as desigualdades nos determinantes sociais de saúde podem aumentar a vulnerabilidade de as pessoas desenvolverem hanseníase e apresentarem incapacidades físicas decorrentes da doença 25 , bem como evoluírem para óbito pela incapacidade física e/ou por complicações sistêmicas 8 .

A tendência de incremento de óbitos por hanseníase também foi observada em municípios com população abaixo de 50 mil habitantes. Tais municípios podem contribuir para o aumento do coeficiente de detecção, bem como para o aumento do número de óbitos decorrentes da doença. Pequenas populações podem compor com áreas de risco para detecção de casos novos e mortes por hanseníase, possivelmente devido a fatores relacionados ao acesso aos serviços de saúde, desempenho e descentralização insuficiente das ações de controle da doença 30,31 , com distanciamento dos referenciais de integralidade.

A distribuição da mortalidade relacionada à hanseníase se deu de maneira heterogênea no Estado do Piauí, com destaque para grupos populacionais e regiões de maior vulnerabilidade social, como verificado para o país 8 .

O presente estudo apresenta limitações relativas aos métodos concernentes ao uso de bases de dados secundários, que em geral está vinculado a incompletude e a inconsistência na quantidade e qualidade das informações ao longo do tempo entre os municípios do Estado do Piauí. Tal fato pode justificar a possibilidade de subestimativa dos achados relatados neste estudo. Outro fator de limitação do estudo está associado ao fato de o óbito por hanseníase ser um evento raro, de modo que se torna fundamental a investigação epidemiológica por meio de outras bases de dados para embasar os dados disponíveis. Vale destacar que a doença não está associada classicamente ao óbito e que os próprios registros do Sistema de Informações sobre Agravos de Notificação (SINAN) não possuem a opção de óbito por hanseníase, o que pode estar comprometendo a avaliação e o registro de óbitos entre os casos que tiveram alta por cura. Entretanto, um estudo com série histórica de 16 anos em um estado de alta endemicidade para hanseníase no país, com poucas evidências de base populacional disponibilizadas, justifica a utilização da base de dados supracitada.

\section{Conclusão}

A mortalidade por hanseníase no Estado do Piauí persiste como problema significativo de saúde pública espacialmente heterogêneo e temporalmente crescente. A análise espacial destaca áreas de concentração com altos coeficientes de mortalidade, em regiões que apresentam altos coeficientes de deteç̧ão de casos novos da doença, além de elementos de vulnerabilidade social e programática.

As tendências temporais de aumento verificadas em áreas e populações bem definidas (homens, idosos, raça/cor parda ou preta e residentes em municípios com menos de 20 mil habitantes) demandam o fortalecimento de ações de vigilância e controle ainda mais estratégicas, tendo o óbito relacionado à hanseníase como evento sentinela. Portanto, reforça-se a importância do desenvolvimento das ações de vigilância de óbitos dentro da rede de atenção à saúde, tendo em vista que a longitudinalidade do cuidado é um atributo inerente à atenção primária à saúde que se responsabiliza pelo acompanhamento dos usuários ao longo do tempo, especialmente em condições crônicas com elevada carga de endemicidade, de modo que se torna imprescindível a atuação da atenção primária à saúde por meio da qualificação das ações de vigilância e controle em hanseníase. 


\section{Colaboradores}

O. D. Araújo, A. F. Ferreira e A. N. Ramos Jr. contribuíram com a concepção e o delineamento do estudo, análise e interpretação dos dados, redação do artigo, revisão crítica relevante do conteúdo intelectual e aprovação final da versão a ser publicada. T. M. E. Araújo, L. C. L. Silva, W. M. P. S. Lopes e J. A. Cardoso colaboraram com a análise e interpretação dos dados, redação do artigo, revisão crítica relevante do conteúdo intelectual e aprovação final da versão a ser publicada. E. A. Rodrigues, N. J. M. Costa, E. H. Moura, S. M. G. Bezerra e M. S. Macêdo contribuíram com a análise e interpretação dos dados, revisão crítica relevante do conteúdo intelectual e aprovação final da versão a ser publicada.

\section{Informações adicionais}

ORCID: Olívia Dias de Araújo (0000-0002-99744338); Anderson Fuentes Ferreira (0000-00021816-9459); Telma Maria Evangelista de Araújo (0000-0001-5628-9577); Laila Caroline Leme da Silva (0000-0003-3516-8658); Walquirya Maria Pimentel Santos Lopes (0000-0001-8589-3132); Érica Alencar Rodrigues Neri (0000-0003-40217194); Jonas Alves Cardoso (0000-0002-55682024); Joelma Maria Costa (0000-0003-2899-9533); Edmércia Holanda Moura (0000-0001-5843-8740); Sandra Marina Gonçalves Bezerra (0000-00033890-5887); Michelle Santos Macêdo (0000-00015676-928X); Alberto Novaes Ramos Jr. (00000001-7982-1757).

\section{Agradecimentos}

A. F. Ferreira é bolsista de mestrado do Conselho Nacional de Desenvolvimento Científico e Tecnológico (CNPq). A. N. Ramos Jr. é bolsista de produtividade em pesquisa do CNPq/Ministério da Ciência, Tecnologia, Inovações e Comunicações.

\section{Referências}

1. Britton WJ, Lockwood DN. Leprosy. Lancet 2004; 363:1209-19.

2. Rocha MCN, Lima RB, Stevens A, Gutierrez MMU, Garcia LP. Óbitos registrados com causa básica hanseníase no Brasil: uso do relacionamento de bases de dados para melhoria da informação. Ciênc Saúde Colet 2015; 20:101726.

3. Moura RS, Penna GO, Cardoso LPV, Pontes MAA, Cruz R, Gonçalves HS, et al. Description of leprosy classification at baseline among patients enrolled at the uniform multidrug therapy clinical trial for leprosy patients in Brazil. Am J Trop Med Hyg 2015; 92:1280-4.

4. World Health Organization. Global leprosy update, 2018: moving towards a leprosy-free world. Wkly Epidemiol Rec 2019; 94:389-412.

5. Secretaria de Vigilância em Saúde, Ministério da Saúde. Caracterização da situação epidemiológica da hanseníase e diferenças por sexo, Brasil, 2012-2016. Boletim Epidemiológico 2018; 49:1-12.

6. IntegraHans PI. Boletim de vigilância em saúde do Estado do Piauí: hanseníase 2016. Teresina: Universidade Federal do Piauí; 2016.

7. Souza EA, Boigny RN, Oliveira HX, Oliveira MLW, Heukelbach J, Alencar CH, et al. Tendências e padrões espaço-temporais da mortalidade relacionada à hanseníase no Estado da Bahia, Nordeste do Brasil, 1999 a 2014. Cad Saúde Colet (Rio J.) 2018; 26:191-202.

8. Martins-Melo FR, Assunção-Ramos AV, Ramos Jr. AN, Alencar CH, Montenegro Jr. RM, Wand-Del-Rey de Oliveira ML, et al. Leprosyrelated mortality in Brazil: a neglected condition of a neglected disease. Trans R Soc Trop Med Hyg 2015; 109:643-52.

9. Martins-Melo FR, Ramos Jr. AN, Alencar CH, Heukelbach J. Mortality from neglected tropical diseases in Brazil, 2000-2011. Bull World Health Organ 2016; 94:103-10.

10. Martins-Melo FR, Carneiro M, Ramos Jr. AN, Heukelbach J, Ribeiro ALP, Werneck GL. The burden of neglected tropical diseases in Brazil, 1990-2016: a subnational analysis from the Global Burden of Disease Study 2016. PLoS Negl Trop Dis 2018; 4:e0006559.

11. Martins-Melo FR, Ramos Jr. AN, Alencar CH, Heukelbach J. Trends and spatial patterns of mortality related to neglected tropical diseases in Brazil. Parasite Epidemiol Control 2016; 1:56-65.

12. World Health Organization. International statistical classification of diseases and related health problems. 10th Rev. Geneva: World Health Organization; 2011.

13. Campos MRM, Batista AVA, Guerreiro JV. Perfil clínico-epidemiológico dos pacientes diagnosticados com hanseníase na Paraíba e no Brasil, 2008-2012. Rev Bras Ciênc Saúde 2018; 22:79-86. 
14. Levorato CD, Mello LM, Silva AS, Nunes AA. Fatores associados à procura por serviços de saúde numa perspectiva relacional de gênero. Ciênc Saúde Colet 2014; 19:1263-74.

15. Souza EA, Ferreira AF, Boigny RN, Alencar CH, Heukelback J, Martins-Melo FR, et al. Leprosy and gender in Brazil: trends in na endemic area of the Northeast region, 2001-2014. Rev Saúde Pública 2018; 52:20.

16. Kerr-Pontes LRS, Barreto ML, Evangelista CMN, Rodrigues LC, Heukelbach J, Feldmeier $\mathrm{H}$. Socioeconomic, environmental, and behavioural risk factors for leprosy in North-east Brazil: results of a case-control study. Int J Epidemiol 2006; 35:994-1000.

17. Gómez L, Rivera A, Vidal Y, Bilbao J, Kasang C, Parisi S, et al. Factors associated with the delay of diagnosis of leprosy in north-eastern Colombia: a quantitative analysis. Trop Med Int Health 2018; 23:193-98.

18. Ferreira AF, Souza EA, Lima MS, Garcia GSM, Corona F, Andrade ESN, et al. Mortalidade por hanseníase em contextos de alta endemicidade: análise espaço-temporal integrada no Brasil. Rev Panam Salud Pública 2019; 43:e87.

19. Lopes VAS, Rangel EM. Hanseníase e vulnerabilidade social: uma análise do perfil socioeconômico de usuários em tratamento irregular. Saúde Debate 2014; 38:817-29.

20. Walker SL, Lebas E, Doni SN, Lockwood DNJ, Lambert SM. The mortality associated with erythema nodosum leprosum in Ethiopia: a retrospective hospital-based study. PLoS Negl Trop Dis 2014; 8:e2690.

21. Rocha MCN, Garcia LP. Investigação epidemiológica dos óbitos notificados tendo como causa básica a hanseníase, ocorridos em Fortaleza, Ceará, 2006-2011. Epidemiol Serv Saúde 2014; 23:277-86.

22. Nogueira PSF, Marques MB, Coutinho JFV, Maia JC, Silva MJ, Moura ERF. Factors associated with the functional capacity of older adults with leprosy. Rev Bras Enferm 2017; 70:711-8.
23. Matos TS, Carmo RF, Santos FGB, Souza CDF. Leprosy in the elderly population and the occurrence of physical disabilities: is there cause for concern? An Bras Dermatol 2019; 94:243-5.

24. Silva DDB, Tavares CM, Gomes NMC, Cardoso AC, Arcênio RA, Nogueira PSF. A hanseníase na população idosa de Alagoas. Rev Bras Geriatr Gerontol 2018; 21:573-81.

25. Heidinger M, Simonnet E, Karippadathu SF, Puchinger M, Pfeifer J, Grisold A. Analysis of social determinants of health and disability scores in leprosy-affected persons in Salem, Tamil Nadu, India. Int J Environ Res Public Health 2018; 15:69.

26. Ribeiro MDA, Silva JCA, Oliveira SB. Estudo epidemiológico da hanseníase no Brasil: reflexão sobre as metas de eliminação. Rev Panam Salud Pública 2018; 42:e42.

27. Carrapato P. Health determinants in Brasil: searching for health equity. Saúde Soc 2017; 26:676-89.

28. Chaves EC, Costa SV, Flores RLR, Neves EOS. Índice de carência social e hanseníase no Estado do Pará em 2013: análise espacial. Epidemiol Serv Saúde 2017; 26:807-16.

29. Boigny RN, Souza EA, Romanholo HSB, Araújo OD, Araújo TME, Carneiro MAG, et al. Persistence of leprosy in household social networks: overlapping cases and vulnerability in endemic regions in Brazil. Cad Saúde Pública 2019; 35:e00105318.

30. Leal DR, Cazarin G, Bezerra LCA, Albuquerque AC, Felisberto E. Programa de controle da hanseníase: uma avaliação da implantação no nível distrital. Saúde Debate 2017; 38:209-28.

31. Saltarelli RMF, Seixas DHT. Limites e possibilidades na atenção ao portador de hanseníase no âmbito da estratégia saúde da família. Rev APS 2016; 19:613-22. 


\section{Abstract}

The study aimed to analyze spatial patterns and time trends in leprosy-related mortality in the State of Piaui, Brazil, from 2000 to 2015. This was a mixed ecological study with a spatial and temporal state-based approach, using data from the Brazilian Mortality Information System. The analysis includes epidemiological characteristics, mortality trends by Joinpoint regression, and spatial analysis, using the state's 224 municipalities (counties) as the geographic unit. Of the 245,413 deaths identified, leprosy was identified in 234 death certificates, 135 (41.7\%) as the underlying cause of death and 189 (58.3\%) as an associated cause of death. The highest leprosy-related mortality rates were associated with male gender (relative risk $-R R=2.38$; 95\%CI: 1.87; 3.03), elderly age $(R R=10.52 ; 95 \%$ CI: 7.16; 15.46), brown skin color $(R R=2.22 ; 95 \% C I: 1.47 ; 3.35)$, and residents of the state's interior $(R R=5.72$; 95\%CI: 4.54; 7.21). The crude leprosy-related mortality rate showed a significant increase among the elderly (70 years), brown race/color, cities with fewer than 20,000 inhabitants, and the Central region of the state, but not significant for the State of Piaui as a whole. The spatial distribution by age-adjusted mortality was heterogeneous in the municipalities, concentrating high mortality rates in the northern region of the state, close to the coastline. There was a pattern of increasing smoothed mortality rates over the course of the study's four-year periods in the Mid-northern Central and Semiarid regions. Leprosy mortality was spatially heterogeneous and growing over the years. The findings highlight the importance of enhancing integrated surveillance and healthcare activities.

Leprosy; Mortality; Spatial Analysis; Time Series Studies

\section{Resumen}

El objetivo del estudio fue analizar patrones espaciales y tendencias temporales de mortalidad, relacionada con la hanseniasis en el Estado de Piauí, Brasil, de 2000 a 2015. Se trata de un estudio ecológico mixto, con un abordaje espacial y temporal, de base estatal, a partir de datos del Sistema de Información sobre Mortalidad. El análisis incluye características epidemiológicas, tendencias de mortalidad por regresión Joinpoint y análisis espacial, usando 224 municipios como unidad geográfica. De los 245.413 óbitos identificados, la hanseniasis fue identificada en 324 declaraciones, 135 (41,7\%) como causa básica de óbito y 189 (58,3\%) como asociada. Los mayores coeficientes de mortalidad relacionada con la hanseniasis se observaron entre hombres (riesgo relativo $-R R=2,38$; IC95\%: 1,87 ; 3,03), ancianos $(R R=10,52$; IC95\%: 7,16; 15,46), color pardo $(R R=2,22$; IC95\%: 1,$47 ; 3,35)$ $y$ residentes en el interior del estado $(R R=5,72$; IC95\%: 4,54; 7,21). El coeficiente bruto de mortalidad relacionado con la hanseniasis presentó un incremento significativo entre ancianos (70 años), raza/color pardo, en ciudades con menos de 20.000 habitantes y región Medio-norte, pero no es significativo para el Estado de Piauí. La distribución espacial por los coeficientes de mortalidad ajustada por edad fue heterogénea en los municipios, concentrando altos coeficientes de mortalidad en el norte del estado, cercano al litoral. Se verificó un patrón de aumento de los coeficientes de mortalidad suavizados en el transcurso de los cuatrienios del estudio, concentrando altos coeficientes en las regiones Medio-norte y Semiárida. La mortalidad por hanseniasis es heterogénea espacialmente y creciente a lo largo de los años. Se resalta la importancia de potenciar acciones integradas de vigilancia y atención a la salud.

Lepra; Mortalidad; Análisis Espacial; Estudios de Series Temporales
Recebido em 26/Mai/2019

Versão final reapresentada em 24/Jan/2020 Aprovado em 13/Fev/2020 